\section{Mortalidad por enfermedad de Parkinson en Chile}

\author{
PEDRO CHANÁ C. ${ }^{1,5}$, MAGDALENA JIMÉNEZ C. ${ }^{a}$, \\ VIOLETA DÍAZ T. ${ }^{4}$, CARLOS JURI ${ }^{2,3}$
}

\section{Parkinson disease mortality rates in Chile}

Background: Parkinson disease (PD) is the second more common neurodegenerative disorder and determines a loss in quality of life, caregiver burden and increased mortality in those affected. Aim: To determine the rates of mortality due to Parkinson disease in Chile. Material and Methods: Data were collected from death certificates between 1997 and 2008. Mortality rates were calculated and the mortality trend was established along the study period, and analyzed by age, sex and geographic region of Chile. Results: There was a steady increase in mortality over the years, particularly since $2001\left(r^{2}=0.85, p<0.01\right)$. The increase in mortality rates was 0.25 per 100.000 habitants/year $\left(r^{2}: 0.89, p<0.01\right)$. Mortality was always higher in men and in those older than 80 years. The highest rate (per 100,000 habitants) in Chile was observed in the region of Valparaiso (2.4) while the lowest was in Antofagasta $(0.8)(t=3.72$, $p<0.05)$. Conclusions: Mortality associated with PD has increased progressively, consistent with the expected increase in prevalence of the disease in the population.

(Rev Med Chile 2013; 141: 327-331).

Key words: Cause of death; Eidemiology; Parkinson disease.

\author{
${ }^{1}$ Centro de Trastornos del \\ Movimiento (CETRAM). \\ Universidad de Santiago de \\ Chile. Santiago, Chile. \\ ${ }^{2}$ Departamento de \\ Neurología. Pontificia \\ Universidad Católica de \\ Chile. Santiago, Chile. \\ ${ }^{3}$ Servicio de Neurología. \\ Hospital Dr. Sótero del Río. \\ Santiago, Chile. \\ ${ }^{4}$ Departamento de \\ Neurología. Hospital Clínico \\ Universidad de Chile. \\ ${ }^{5}$ Clínica Alemana de \\ Santiago. \\ anterna de Medicina. \\ Pontificia Universidad \\ Católica de Chile. \\ Recibido el 23 de abril de \\ 2012, aceptado el 3 de \\ octubre de 2012. \\ Correspondencia a : \\ Carlos Juri Clavería \\ Departamento de \\ Neurología. Facultad de \\ Medicina. \\ Pontificia Universidad \\ Católica de Chile. \\ Marcoleta 350. Segundo \\ Piso. Santiago. Chile. \\ 56-2-3543316 \\ E-mail: cjuri@med.puc.cl
}

L a enfermedad de Parkinson (EP) es una condición neurodegenerativa de curso progresivo. La EP determina un deterioro de la calidad de vida de los pacientes, sobrecarga para los familiares y cuidadores y un aumento de la mortalidad de los afectados ${ }^{1}$. Aproximadamente 1 a $2 \%$ de la población mayor de 65 años sufre EP, cifra que se eleva a 3\%-5\% en aquellos mayores de 85 años ${ }^{2}$. La prevalencia en países europeos es entre 100 y 200 por cada 100.000 habitantes. Las tasas de incidencia varían entre los estudios, probablemente por aspectos metodológicos, la tasa de incidencia, estandarizada por edad, reportada en Europa y Estados Unidos de Norteamérica varía entre 8,6 y 19,0 por cada 100.000 habitantes $^{2}$. Se estima que en el mundo existen cerca de 4 millones de pacientes afectados actualmente por esta condición $^{3}$ y se espera un aumento progresivo del número de individuos afectados, producto del aumento de la sobrevida de la población, en particular de los países en desarrollo ${ }^{3}$.

En nuestro país, no existen datos precisos sobre la prevalencia de esta condición, sólo un estudio ha estimado el número de afectado, pero su metodología dificulta hacer estimaciones adecuadas ${ }^{4}$. Basados en datos internacionales se puede estimar que existen cerca de 40.000 pacientes con EP en Chile 3 . Conocer el número de personas afectadas por una enfermedad es importante para diseñar adecuadamente los cuidados y destinar los recursos económicos y humanos correspondientes ${ }^{1,5}$. Este estudio tiene como objetivo evaluar la tendencia de mortalidad por EP en Chile en un período de 10 años y valorar las variaciones geográficas de dicha mortalidad.

\section{Métodos}

Se estudió la tendencia de la tasa de mortalidad general, por rango de edad y sexo, asociada a EP, durante el período 1997-2008. Los datos se obtuvieron a partir de los anuarios demográficos de mortalidad del ministerio de Salud de Chile, los que son generados a partir de certificados de 
defunción. Se utilizaron las categorías diagnósticas de la Clasificación Internacional de Enfermedades, correspondiente al código 332.0 para el período 1997-2000 (versión 9.0) y G 20 para el período 2001-2008 (versión 10.0). Se consideró los pacientes fallecidos con diagnóstico de EP entre las dos primeras causas de muerte del certificado de defunción.

Se elaboraron tasas por 100.000 habitantes y sus intervalos de confianza al 95\%, para poblaciones totales y divididas por sexo y edad, considerando en el numerador los pacientes fallecidos por EP y en el denominador la población media estimada para cada año en Chile. Se construyeron tasas por regiones, empleando la población media anual de cada región a lo largo del período estudiado. Se utilizó para esto las cifras de la base de datos del Instituto Nacional de Estadísticas (INE) de las proyecciones estimadas de población para los años 1990-2020, basadas en el Censo 2002 y elaboradas a nivel nacional, regional, provincial y comunal y se construyó un mapa nacional de tasas por regiones clasificando las tasas regionales en 5 categorías.

Para estimar la tendencia de mortalidad en el tiempo, las tasas de mortalidad fueron estandarizadas por método directo por edad usando la población estándar de la Organización Mundial de la Salud (OMS) (rangos: $(<50,50-59,60-69$, 70-79, > 80).

El estudio de tendencia se efectuó mediante análisis de correlación de Pearson y para el cálculo de pendiente se empleó regresión lineal. La comparación de tasas se realizó mediante t-test. Se estableció un nivel de significación estadística de $\mathrm{p}<0,05$.

\section{Resultados}

Se detectaron 3.619 fallecimientos con mención de EP en el período 1997-2008. De ellos 1.741 casos correspondieron a mujeres $(48,1 \%)$. La tasa de mortalidad cruda observada (por 100.000 habitantes) en la población general mostró un crecimiento significativo en el período, aumentando desde 0,79 en 1997 hasta 3,0 en $2008\left(r^{2}=0,85\right.$, $\mathrm{p}<0,0001)$, mientras que la tasa ajustada por edad aumentó de 3,1 a 11,0 $\left(\mathrm{r}^{2}=0,91, \mathrm{p}<0,0001\right)$, (Figura 1 y Tabla 1 ).

Se observó predominio del sexo masculino en todo el período estudiado (Figuras 1 y 2) y en todos los grupos de edad analizados, en particular en sujetos de mayor edad (Figuras 2 y 3 ), aunque esta diferencia sólo alcanzó significación estadística al comparar tasas ajustadas $(\mathrm{p}=0,04)$. La tasa de mortalidad mostró una tendencia creciente significativa en ambos sexos. En hombres, la tasa cruda aumentó de 0,87 en 1997 a 3,25 en 2008 $\left(r^{2}=0,89, p<0,0001\right)$, mientras que en mujeres varió de 0,72 a $2,85\left(\mathrm{r}^{2}=0,90, \mathrm{p}<0,0001\right)(\mathrm{Ta}-$ bla 2). La mortalidad asociada a EP fue mayor con el aumento de la edad de la población, alcanzando tasas específicas hasta 100 por 100.000 habitantes en sujetos mayores de 80 años, significativamente mayores a lo observados en pacientes menores de 60 años $(\mathrm{p}<0,001)$.

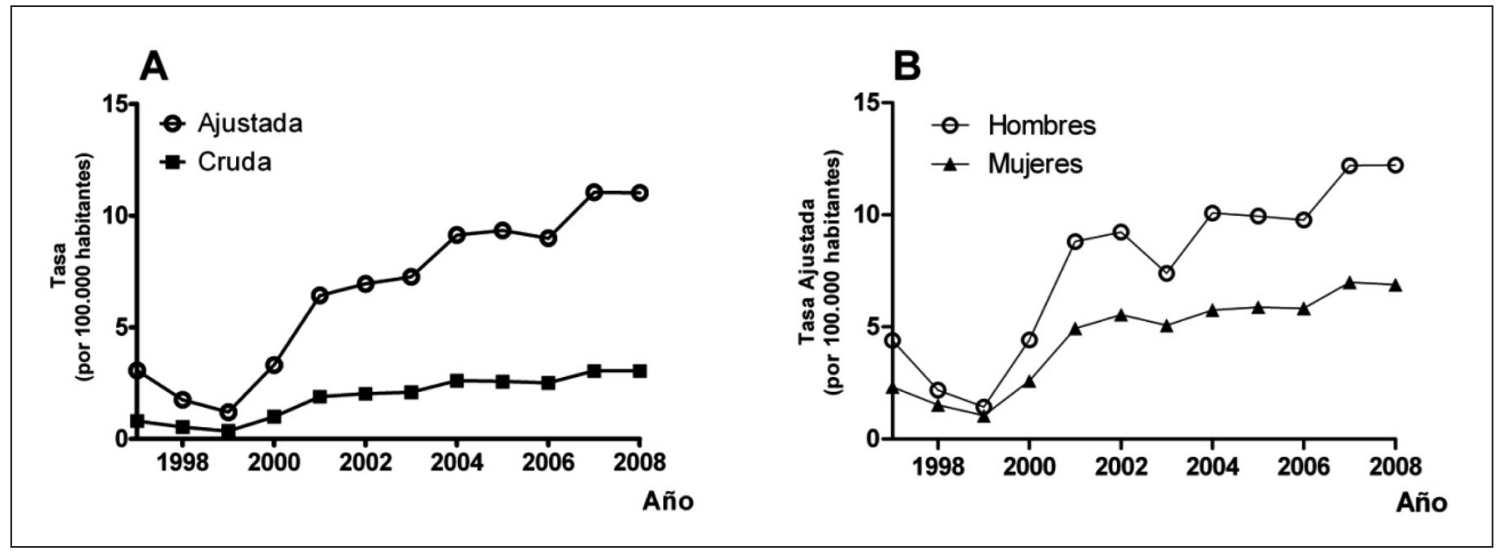

Figura 1. Mortalidad por Enfermedad de Parkinson en Chile. Se presenta la evolución de la tasa de mortalidad cruda y ajustada por edad asociada e E.P. (A) y según sexo (B) reportada en los certificados de defunción en el período 1997-2008. Valores expresados por cada 100.000 habitantes. 
Mortalidad por enfermedad de Parkinson en Chile - P. Chaná et al

Tabla 1. Tasa de mortalidad por EP en Chile durante el período 1997-2008

\begin{tabular}{|cccc|}
\hline Año & $\begin{array}{c}\text { Tasa mortalidad } \\
\text { (por 100.000 } \\
\text { habitantes) }\end{array}$ & \multicolumn{2}{c|}{$\begin{array}{c}\text { Intervalo de } \\
\text { confianza (95\%) }\end{array}$} \\
\hline 1997 & 0,80 & 0,65 & 0,94 \\
1998 & 0,53 & 0,42 & 0,65 \\
\hline 1999 & 0,36 & 0,27 & 0,46 \\
\hline 2000 & 0,99 & 0,83 & 1,14 \\
\hline 2001 & 1,89 & 1,68 & 2,11 \\
\hline 2002 & 2,03 & 1,80 & 2,25 \\
\hline 2003 & 2,09 & 1,86 & 2,31 \\
\hline 2004 & 2,61 & 2,36 & 2,86 \\
\hline 2005 & 2,57 & 2,32 & 2,82 \\
\hline 2006 & 2,51 & 2,27 & 2,76 \\
\hline 2007 & 3,05 & 2,78 & 3,31 \\
\hline 2008 & 3,05 & 2,78 & 3,31 \\
\hline
\end{tabular}

Tabla 2. Análisis de pendiente de la tendencia de la mortalidad por enfermedad de Parkinson, durante el período 1997-2008

\begin{tabular}{|lccc|}
\hline Año & $\begin{array}{c}\text { Pendiente tasa } \\
\text { mortalidad } \\
\text { ( } \pm \text { EE) }\end{array}$ & $\mathbf{r}^{\mathbf{2}}$ & Valor-p \\
Población general & $0,25 \pm 0,03$ & 0,89 & $<0,0001$ \\
Hombres & $0,27 \pm 0,03$ & 0,89 & $<0,0001$ \\
Mujeres & $0,24 \pm 0,02$ & 0,90 & $<0,0001$ \\
\hline
\end{tabular}

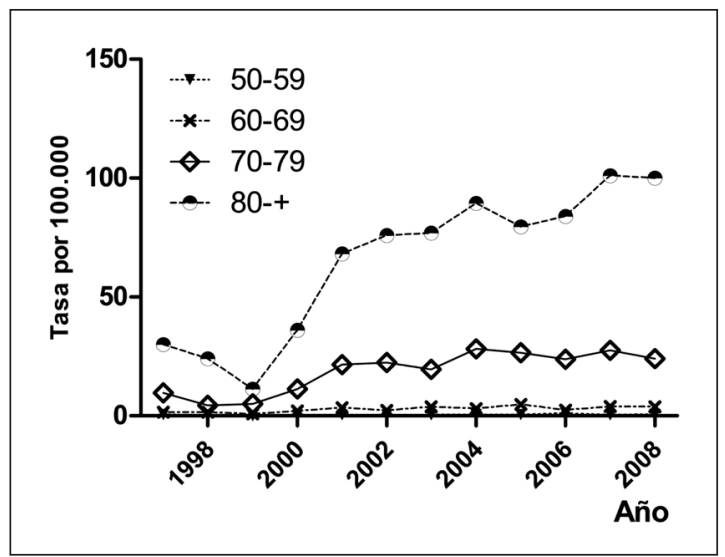

Figura 2. Tasa de mortalidad por edad por Enfermedad de Parkinson en Chile, por cada 100.000 habitantes.

La edad de muerte en los hombres fue (media \pm DE) $78,3 \pm 8,6$ años y en las mujeres $80,4 \pm 8,7$ años $(\mathrm{p}<0,001 ; \mathrm{t}=-5,45)$.

Finalmente, se evaluó la mortalidad por EP en las diferentes regiones de Chile en el período estudiado. Se observó diferencias en las tasas entre las distintas áreas. En particular, la menor tasa promedio de mortalidad específica (por cada 100.000 habitantes) se detectó en la segunda región $(0,95$ $\pm 0,68)$ y la más alta en la quinta región $(2,41 \pm$ $1,2)$, con diferencia estadísticamente significativa entre ambas $(t=3,72, p<0,05)$ con una tasa cruda promedio país de todo el período estudiado de $1,71 \pm 0,79$ (Figura 4).

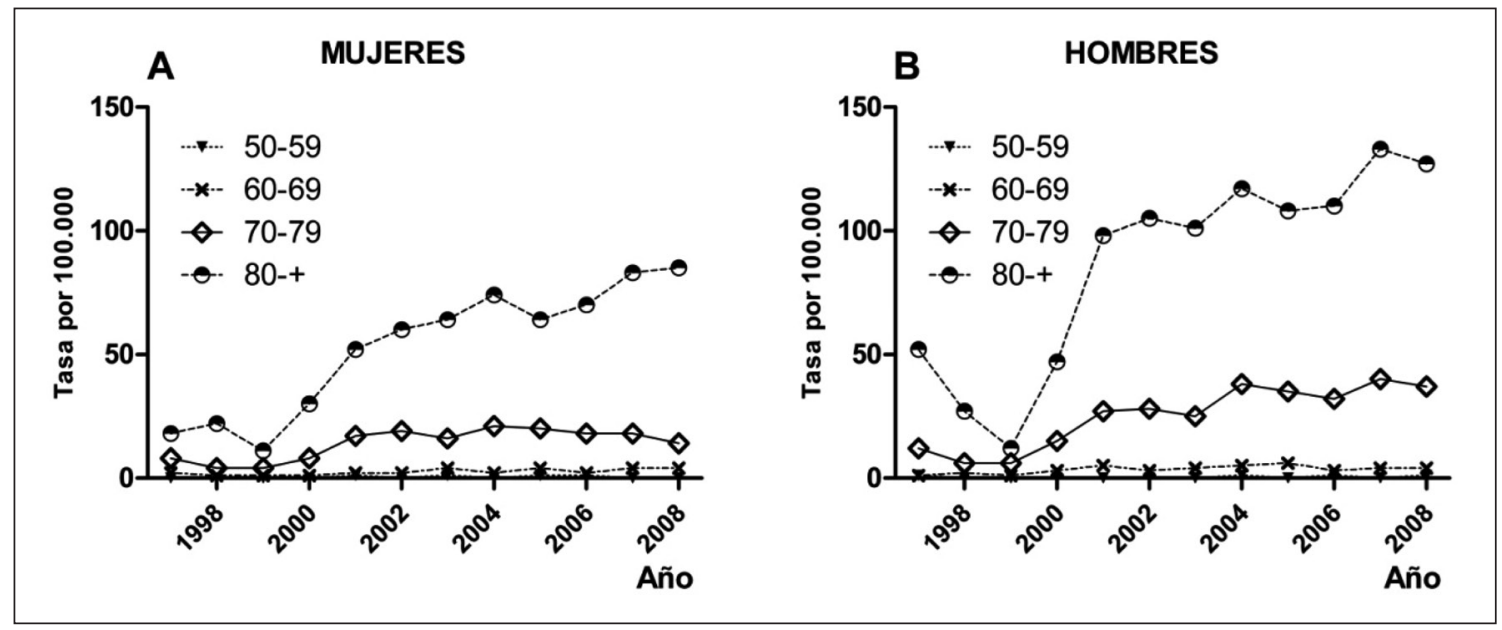

Figura 3. Tasa de mortalidad específica por Enfermedad de Parkinson en Chile por edad y según sexo por cada 100.000 habitantes. 


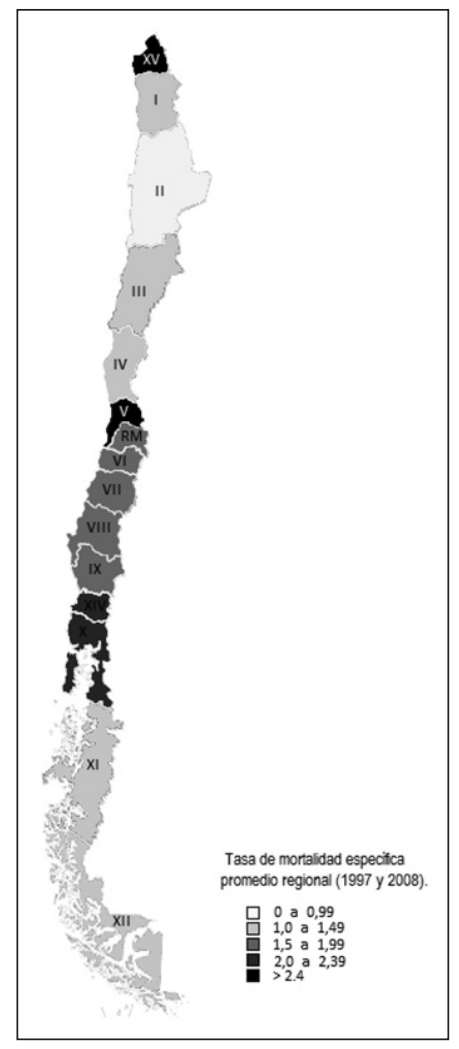

Figura 4. Tasa de mortalidad específica promedio, asociada a enfermedad de Parkinson, en las distintas regiones entre los años 1997 y 2008 Valores expresados por cada 100.000 habitantes.

\section{Discusión}

La EP es una condición neurodegenerativa crónica de causa desconocida en la mayoría de los casos, que genera disminución en la calidad de vida de los pacientes y sobrecarga de sus cuidadores ${ }^{5}$, además de acortar la expectativa de vida de quienes la padecen ${ }^{6}$.

En nuestra serie, la única reportada hasta la fecha sobre mortalidad en EP en población chilena, se ha recolectado los certificados de defunción a lo largo de los últimos 11 años, recogiéndose un total de más de 3.500 casos de EP mencionados como primera o segunda causa de defunción. Nuestros resultados muestran una tendencia creciente de la EP como causa de mortalidad en nuestra población.

El análisis de los datos empleando tasas corregidas directamente por edad a lo largo del tiempo, para hacer comparables las distintas muestras entre sí, arroja un discreto descenso entre los años 1997 y 1999, con una tendencia de un marcado aumento progresivo a partir del año 2000. Esta variación de la tendencia coincide con el cambio del sistema de registro de las enfermedades propuesto por la Organización mundial de la salud (OMS), en particular con el cambio de la versión CIE-9 a la versión CIE-10. Otros estudios han mostrado que en EP la clasificación utilizada es un factor determinante de la frecuencia de reportes ${ }^{7-9}$. No obstante lo anterior, es posible observar una tendencia sostenida al alza de EP en los certificados de defunción, lo que sugiere un aumento real de la prevalencia de esta condición en los fallecidos.

El análisis por sexos demuestra el predominio del reporte de mortalidad por EP en hombres, siendo su tasa promedio por 100.000 habitantes $7,3 \%$ más frecuente comparada con mujeres, lo que se observa en todos los grupos etáreos analizados y coincide con la historia natural de la $\mathrm{EP}^{10}$. También se demuestra que la muerte en hombres portadores de EP ocurre en promedio 1,5 años antes que en mujeres, lo que coincide con la menor esperanza de vida de los hombres en nuestra población.

El análisis por regiones demuestra la presencia de tasas de mortalidad menores en regiones del norte grande y del extremo sur, concentrándose la mayor mortalidad en áreas del centro y sur del país. Varios estudios epidemiológicos han asociado el desarrollo de EP a la vida rural ${ }^{11}$, desconocemos en nuestra población estudiada la procedencia rural o urbana como para establecer dicha relación, además que no es posible a partir de datos de mortalidad estimar directamente la incidencia de esta patología. Sin embargo, las notables variaciones en la mortalidad pueden indicar diferencias en el reporte o en el tratamiento de estos pacientes ${ }^{12}$.

Los estudios sobre mortalidad basados en certificados de defunción presentan numerosas dificultades metodológicas ya que descansan en diagnósticos establecidos por médicos no especialistas en todas las patologías, quienes a veces cuentan con información incompleta sobre la historia médica del paciente ${ }^{13,14}$. En particular, en la enfermedad de Parkinson diferentes trabajos han mostrados reportes entre 60 y $75 \%$ de esta enfermedad en los certificados de defunción de pacientes con diagnóstico establecido adecuadamente y en conocimiento de la familia, lo que demuestra un índice de sub-reporte importante ${ }^{15,16}$. No obstante, la información contenida en los certificados de defunción ha sido utilizada ampliamente para establecer el comportamiento de distintas enfer- 
Mortalidad por enfermedad de Parkinson en Chile - P. Chaná et al

medades crónicas y agudas y para monitorizar su curso temporal y su comportamiento geográfico, por lo tanto, reconociendo las limitaciones propias de esta metodología, es posible extraer resultados orientativos sobre las tendencias de algunas enfermedades que permitan obtener información útiles sobre aspectos patogénicos y terapéuticos a tener en cuenta ${ }^{17}$.

Nuestros resultados demuestran un aumento de la tasa de mortalidad por EP a lo largo del período estudiado, que puede estar relacionado al cambio de la clasificación internacional de las enfermedades o al reporte por parte de los profesionales, sin embargo, no podemos descartar que exista un real aumento de la mortalidad por EP en el período estudiado ${ }^{8}$. También demostramos diferencias en la tasa de mortalidad por regiones cuyas causas son poco claras. Nosotros planteamos que nuestros datos enfatizan la necesidad de realizar estudios epidemiológicos prospectivos en esta población de pacientes, que permitan establecer la incidencia de esta enfermedad a lo largo de nuestro país y determinar los factores que condicionen su variación a lo largo de nuestra geografía y que permitan esclarecer los determinantes de la mortalidad de los pacientes con $\mathrm{EP}^{12}$.

\section{Referencias}

1. Asimakopoulos P, Caslake R, Harris C, Gordon J, Taylor $\mathrm{K}$, Counsell C. Changes in quality of life in people with Parkinson's disease left untreated at diagnosis. J Neurol Neurosurg Psychiatry 2008; 79: 716-8.

2. Alves G, Forsaa E, Pedersen K, Dreetz Gjerstad M, Larsen J. Epidemiology of Parkinson's disease. J Neurol 2008; 255: 18-32.

3. Dorsey E, Constantinescu R, Thompson J, Biglan K, Holloway R, Kieburtz K, et al Projected number of people with Parkinson disease in the most populous nations, 2005 through 2030. Neurology 2007; 68: 384-6.

4. Chiofallo N, Kirschbaum A, Schoenberg B, Olivares O, Soto E. Estudio epidemiológico de las enfermedades neurológicas en Santiago Metropolitano, Chile. Rev Neuro Psiquiat 1992; 30: 335-41.
5. Martínez-Martín P, Arroyo S, Rojo-Abuin J, RodríguezBlazquez C, Frades B, de Pedro Cuesta J, et al. Burden, perceived health status, and mood among caregivers of Parkinson's disease patients. Mov Disord 2008; 23: 1673 80.

6. Ishihara L, Cheesbrough A, Brayne C, Schrag A. Estimated life expectancy of Parkinson's patients compared with the UK population. J Neurol Neurosurg Psychiatry 2007; 78: 1304-9.

7. Bray F, Guilloux A, Sankila R, Parkin D. Practical implications of imposing a new world standard population. Cancer causes \& control 2002; 13: 175-82.

8. Griffiths, C. Rooney C. Trends in mortality from Alzheimer's disease, Parkinson's disease and dementia, England and Wales, 1979-2004. Health Stat Q 2006; 30: 6-14.

9. Richardson D. The impact on relative risk estimates of inconsistencies between ICD-9 and ICD-10. Occup Environ Med 2006; 63: 734-40.

10. Poewe W. The natural history of Parkinson's disease J Neurol 2006; 253: 2-6.

11. Vanacore N, Bonifati, V, Bellatreccia A, Edito F, Meco G et al. Mortality rates for Parkinson's disease and parkinsonism in Italy (1969-1987). Neuroepidemiology 1992; 11: 65-73.

12. Wirdefeldt K, Adami H, Cole P, Trichopoulos D, Mandel J. Epidemiology and etiology of Parkinson's disease: a review of the evidence. Eur J Epidemiol 2011; 26: S1-58.

13. Paulson G, Gill W. Are death certificates reliable to estimate the incidence of Parkinson's disease? Mov Disord 1995; 10: 678.

14. Núñez F, Icaza N. Calidad de las estadísticas de mortalidad en Chile, 1997-2003. Rev Med Chile 2006; 134: 1191-6.

15. Pressley J, Tang M, Marder K, Cote J, Mayeux R. Disparities in the recording of Parkinson's disease on death certificates. Mov Disord 2005; 20: 315-21.

16. Papapetropoulos S, Villar J, González J, Mash D. Disparities in death certificates of Parkinson's disease patients: a report from a population of brain donors. Mov Disord 2006; 21: 1791-2.

17. Phillips N, Reay J, Martyn C. Validity of mortality data for Parkinson's disease. J Epidemiol Community Health 1999; 53: 587-8. 